\title{
Effects of the Purkinje System and Cardiac Geometry on Biventricular Pacing: A Model Study
}

\author{
Daniel Romero, ${ }^{1,2}$ Rafael Sebastian, ${ }^{1,2}$ Bart H. Bijnens, ${ }^{1,2,3}$ Viviana Zimmerman, ${ }^{1,2}$ \\ Patrick M. Boyle, ${ }^{4}$ Edward J. Vigmond, ${ }^{4}$ and Alejandro F. Frangi ${ }^{1,2,3}$ \\ ${ }^{1}$ Computational Imaging \& Simulation Technologies in Biomedicine (CISTIB), Universitat Pompeu Fabra, Carrer Tanger, \\ 122-140 (Office N 55,123), 08018 Barcelona, Spain; ${ }^{2}$ Networking Biomedical Research Center on Bioengineering, Biomaterials \\ and Nanomedicine (CIBER-BBN), Barcelona, Spain; ${ }^{3}$ Institució Catalana de Recerca i Estudis Avançats (ICREA), Barcelona, \\ Spain; and ${ }^{4}$ Department of Electrical and Computer Engineering, University of Calgary, Calgary, AB T2N 1N4, Canada
}

(Received 25 August 2009; accepted 7 January 2010)

Associate Editor Larry V. McIntire oversaw the review of this article.

\begin{abstract}
Heart failure leads to gross cardiac structural changes. While cardiac resynchronization therapy (CRT) is a recognized treatment for restoring synchronous activation, it is not clear how changes in cardiac shape and size affect the electrical pacing therapy. This study used a human heart computer model which incorporated anatomical structures such as myofiber orientation and a Purkinje system (PS) to study how pacing affected failing hearts. The PS was modeled as a tree structure that reproduced its retrograde activation feature. In addition to a normal geometry, two cardiomyopathies were modeled: dilatation and hypertrophy. A biventricular pacing protocol was tested in the context of atrio-ventricular block. The contribution of the PS was examined by removing it, as well as by increasing endocardial conductivity. Results showed that retrograde conduction into the PS was a determining factor for achieving intraventricular synchrony. Omission of the PS led to an overestimate of the degree of electrical dyssynchrony while assessing CRT. The activation patterns for the three geometries showed local changes in the order of activation of the lateral wall in response to the same pacing strategy. These factors should be carefully considered when determining lead placement and optimizing device parameters in clinical practice.
\end{abstract}

Keywords-Cardiac geometry, Electrophysiology, Purkinje system, Heart modeling, Cardiac resynchronization therapy, ventricular pacing.

\section{INTRODUCTION}

Cardiac resynchronization therapy (CRT) is a pacemaker treatment for heart failure patients (New York Heart Association class III and IV). It improves systolic

\footnotetext{
Address correspondence to Daniel Romero, Computational Imaging \& Simulation Technologies in Biomedicine (CISTIB), Universitat Pompeu Fabra, Carrer Tanger, 122-140 (Office N 55,123), 08018 Barcelona, Spain. Electronic mail: daniel.romero@upf.edu
}

function in ventricular dyssynchrony, ${ }^{13}$ leading to amelioration of functional capacity, inverse remodeling, and reduction of morbidity and mortality. $1,5,13$ Despite these benefits, clinical trials have demonstrated that around one third of patients do not respond favorably to CRT using standard clinical selection criteria. Sub-optimal resynchronization resulting from current pacing protocols has been proposed as a potential cause underlying treatment failure. ${ }^{1,6}$

As the use of whole heart computational models for electrophysiological simulations becomes more feasible, ${ }^{31,43}$ they are starting to be considered as a practical way to explore certain hypotheses that are difficult to study in vivo. Various computational studies have undertaken the task of unveiling pathological substrates and assessing treatment methodologies. Current available cardiac models, ranging from single cell, ${ }^{11,26,38}$ to tissue level ${ }^{14,30}$ and organ level, ${ }^{25,42}$ are sufficiently accurate to model complex processes, including ion kinetics in healthy and pathological conditions. In many cases, cardiac modeling can be used to investigate phenomena such as drug effects on the electromechanical response and arrhythmogenesis. ${ }^{14,35}$

Although cardiac geometry can be extracted from existing image modalities, many other important features to the modeling process (i.e., myofiber orientation, tissue conductivity) cannot be noninvasively obtained; thus, population-based data are used instead. Such is the case for the Purkinje fibers, the fast conducting cardiac tissue responsible for synchronous activation of the ventricles during the cardiac cycle. Despite its relevance, its structure and effects are commonly not considered in cardiac simulations. ${ }^{33}$ Previous studies have attempted to model the Purkinje system (PS) following anatomical landmarks based on 
maps of the activation sequence. These maps helped determine roughly the Purkinje myocardial junctions (PMJ) which are contact points for electric impulse transmission between the PS and bulk myocardium. ${ }^{39}$ In some electrical modeling studies, researchers have estimated a time delay function for stimulating a number of nodes on the endocardium to reproduce the depolarizing pattern of the PS. ${ }^{8,42}$ Others have used high-subendocardial conduction velocities to represent the PS influence. ${ }^{18}$ These approaches to incorporating PS functionality through bypassing its specific modeling is problematic because it disables an intrinsic property of the PS: retrograde conduction. Bidirectional electric flow along the PS might not be necessary for normal sinus activation of a healthy heart, but its contribution is important during analysis of CRT, where the electrodes are positioned close to the distal portion of the PS.

Another factor to bear in mind during evaluation of CRT candidates with heart modeling is the geometry of the ventricles. Heart failure patients can suffer from pathological conditions that lead to ventricular remodeling. Such are the cases of dilated cardiomyopathy (DCM), characterized by an increase in ventricular diameter, ${ }^{4}$ and hypertrophic cardiomyopathy (HCM), a thickening of the ventricular wall. $^{34}$

Previous model studies on paced hearts include work focused on the electromechanical effects of pacing each ventricle, ${ }^{17}$ or optimizing CRT pacemaker settings based on electrical information. ${ }^{33}$ The study presented in this paper aims to elucidate changes in the activation pattern due to the interaction of CRT pacing and PS in normal and pathological human geometries. For this purpose, a specific CRT model was built which included a novel and more accurate modeling of the cardiac conduction system than previous approaches. The main advantage of this approach is the possibility to simulate the retrograde activation of the PS resulting from the pacing. Understanding the activation sequence for a particular patient, given the geometrical characteristics and knowing the effect of the underlying structures, could facilitate selection of appropriate treatments and tailor the CRT devices to optimize therapeutic outcome on an individual basis.

The influence of ventricular geometry and PS interaction on the paced heart was studied using three models: (i) a normal patient biventricular (BV) mesh, to which geometrical changes were introduced to emulate (ii) DCM and (iii) HCM. The geometrical models were used to simulate electrical activation under normal sinus rhythm and paced conditions in the context of a third degree atrioventricular (AV) block. Complete blockage of AV conduction leaves the task of ventricular activation to the pacemaker alone.

Electrical propagation in cardiac tissue was modeled using the monodomain formulation. For the PS, an embedded structure was built which allowed to model both Purkinje-Purkinje and Purkinjemyocardium properties independently. For BV pacing, the pacemaker leads were model as current injection points at certain sites of the geometries. A pacing protocol was defined using different sequential pacing strategies (interventricular delays), to understand how histological structure and geometry affects cardiac activation for different pacemaker configurations. For each geometry, each pacing protocol was performed with and without the PS. Furthermore, models with increased conductivity at the endocardium were used to represent a dense PS as described by Kerckhoffs et al. ${ }^{18}$

Section 2 details the construction of the normal BV mesh and the process for applying transformations to arrive at pathologic geometries representing DCM and HCM. Section 3 outlines the mathematical models used to simulate cardiac activity and describes pacing protocols. Section 4 presents the simulation results with a discussion of the most prominent findings and describes the limitations of the modeling approach. Section 5 presents the conclusions from this study.

\section{ANATOMICAL MODEL CONSTRUCTION}

\section{Healthy Anatomy}

A human anatomical model of the right and left ventricles with landmarked endocardial and epicardial surfaces was used in this study. The BV surface mesh was obtained from the segmentation at the end diastolic phase of a cardiac multislice computed tomography (MSCT) scan of a 53-year-old male. A normal anatomy was determined as the subject underwent a diagnostic CT scan. For the patient-specific segmentation, a heart statistical atlas trained and built with 100 MSCT patient scans was used. ${ }^{27}$ An active shape model replaced manual delineations by a model-based method, and enhanced the transfer of a number of functional substructures incorporated in the atlas (i.e., the PS) and anatomical labels. ${ }^{12}$ The models used for the simulations were all BV since general CRT procedures require implanting a pacemaker lead in each ventricle.

\section{Generation of Pathological Anatomies}

The original surface mesh (see Fig. 1a) was mathematically transformed into two new meshes to 
(a)

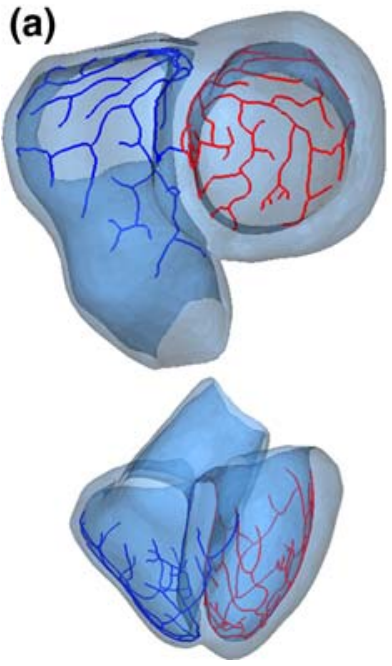

(b)

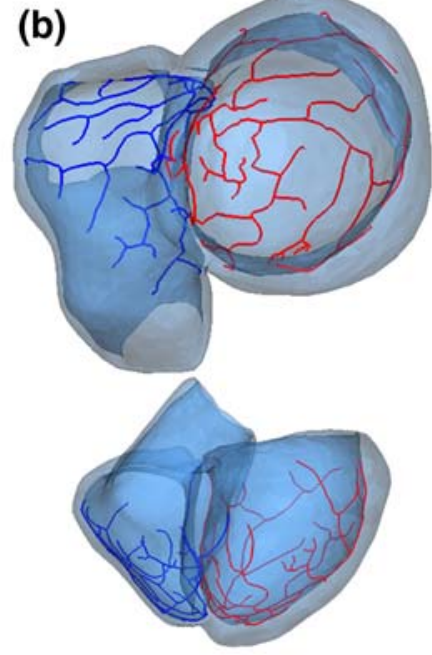

(c)
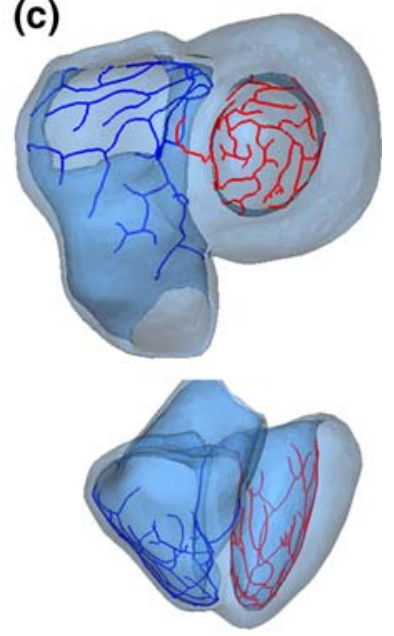

FIGURE 1. Ventricular models. Basal and anterior views of the biventricular surface models: (a) original healthy subject, (b) dilated (DCM), and (c) hypertrophic (HCM) cardiomyopathies. Solid lines represent the RV (blue) and LV (red) branches of the PS.

represent DCM and HCM. In clinical practice, DCM is diagnosed when the ventricular diameter exceeds $117 \%$ of the expected normal, age-adjusted value and HCM is diagnosed when wall thickness exceeds $125 \%$ of the expected maximal thickness. ${ }^{3}$ Based on these criteria and comparing to typical patients, the HCM mesh was constructed by scaling the LV endocardium to achieve a $50 \%$ increase in wall thickness measured radially (see Fig. 1c). For the DCM mesh, the LV endocardial surface was radially displaced to obtain a $50 \%$ increase in diameter. Subsequently, the epicardial surface was further dilated to achieve a $30 \%$ thinning of the average wall thickness. The latter step was done to assure the property of conservation of mass in the myocardium of dilated (nonvolume overloaded) hearts (see Fig. 1b).

\section{Volumetric Meshing}

The three surface meshes were used to create highresolution volumetric tetrahedral meshes needed for numerical simulations. Volumetric mesh quality was ensured by controlling both the maximum distance between neighboring nodes and the radius-to-edge ratio to assure regularly shaped elements. The average inter-node distance was smaller than $500 \mu \mathrm{m}$, with between 2.5 and 3.5 million nodes and between 15 and 21 million linear elements. The conduction velocity at this mesh resolution was tested on a slab of tissue with conductivity values recommended by Clerc. ${ }^{7}$ From this, we obtained a maximum velocity of $0.67 \mathrm{~m} / \mathrm{s}$ which is in the range of reported values. $^{24}$

\section{Myocardial Fiber Orientation}

The fiber orientation was calculated for every element of the mesh, using a mathematical formulation based on the work of Streeter $^{37}$ validated by Jouk et al. ${ }^{15}$ The Helical fiber structure in the Healthy model was computed with fibers rotating from $+50^{\circ}$ at the endocardium to $-60^{\circ}$ at the epicardium. This helical pattern was also used to calculate the fiber orientation for elements in the DCM and HCM meshes. MacGowan et al. demonstrated that anatomic fiber angles were not different between normal subjects and idiopathic DCM patients. ${ }^{20}$ Also Tezuka ${ }^{40}$ concluded from his experimental work that fiber orientation in concentric HCM subjects differs from normal subjects only at the endocardium.

\section{Purkinje System}

The PS was manually delineated as an independent structure in the atlas, and fitted to the healthy subject surface mesh during segmentation. Terminals were positioned to reproduce the activation sequence reported experimentally by Durrer et al. ${ }^{10}$ and more recently by Ramanathan et $\mathrm{al}^{32}$ Paths between terminals were built using splines to form a branching network. The resulting PS model consisted of the bundle branches and 100 segments distributed over the endocardial surface mesh, with no loops in the network. In Fig. 1, the RV (blue) and LV (red) PS are superimposed on the ventricles.

The PS for the pathological meshes was obtained using the previously described transformation algorithms. It was assumed that in the pathologies 
modeled, the number of branches in the PS was unaffected and no terminals were generated or destroyed. This is reasonable, considering that in HCM and DCM myocytes and Purkinje cells do not undergo hyperplasia during ventricular remodeling. ${ }^{3}$

\section{MATHEMATICAL MODELING OF ELECTROPHYSIOLOGY}

Modeling cardiac electrophysiology involves two main steps: first, calculating the underlying variations in ionic concentrations across the cellular membrane at each node; second, determining the dynamics of electrical activity at the tissue level.

The electrophysiological cell models used in this study were the Ten Tusscher-Panfilov model ${ }^{38}$ for the bulk myocardial cells and the DiFrancescoNoble model $^{9}$ for the PS. The maximum sodium conductance was increased by a factor of 3 in the DiFrancesco-Noble model to augment the conduction velocity.

For the propagation of the electrical impulse in the cardiac tissue, the monodomain formulation was used. ${ }^{29}$ Compared to the bidomain formulation, the monodomain ignores the extracellular field contribution, but it has been demonstrated that for simulations such as those undertaken in this study the difference in results can be neglected. ${ }^{31}$ Thus, choosing the monodomain over the bidomain is a matter of computational efficiency since it only requires solving a parabolic equation as opposed to the parabolic plus an elliptic equation. The semi-implicit Crank-Nicholson method was used to update transmembrane potential as described by Vigmond et $a .^{44}$ and Whiteley, ${ }^{45}$ solving a linear system and preserving stability for large time steps.

The modeling of the PS was performed as described by Vigmond and Clements. ${ }^{41}$ The network was constructed from 1D cubic Hermite elements to ensure continuity of current at junctions and bifurcations. Fibers were described as 1D cables that branched at certain positions forming a network structure. The PS was isolated electrically from the myocardium, with the two tissues connected at PMJs, which were modeled as fixed resistances. Each PMJ was coupled to a group of myocardial nodes within a specified radius. For further information, see Vigmond and Clements ${ }^{41}$ and Boyle et al. ${ }^{2}$ Table 1 gives the specific parameters used in the cable model, where $\Omega_{\mathrm{PMJ}}$ is the PMJ resistance and $\Omega_{\mathrm{PPJ}}$ is the resistance between PS segments, $\sigma$ is the intracellular conductivity, and $I_{\mathrm{His}}$ is the current injected into the His bundle to trigger the Purkinje activation.
TABLE 1. Constant values used for the Purkinje cable model.

\begin{tabular}{ll}
\hline Parameter & \multicolumn{1}{c}{ Value } \\
\hline$\Omega_{\text {PMJ }}$ & $27 \mathrm{M} \Omega$ \\
$\Omega_{\text {PPJ }}$ & $100 \mathrm{k} \Omega$ \\
$\sigma$ & $0.024 \mathrm{~S} / \mathrm{m}^{3}$ \\
$I_{\text {His }}$ & $220 \mu \mathrm{A} / \mathrm{cm}^{3}$ \\
\hline
\end{tabular}

All the numerical calculations were performed using the Cardiac Arrhythmia Research Package (CARP) ${ }^{28,44}$ The simulations were run for $230 \mathrm{~ms}$ of the cardiac cycle since only the onset of depolarization was of interest.

\section{Application to Study Electrical Activation in CRT}

To assess the influence of cardiac geometry and the PS, a set of standard sequential pacing protocols were conducted on the three cardiac models. Seven pacing scenarios determined by different time delays between the RV and LV pacing leads (inter-ventricular delay, or VVD) were tested. The time delays ranged from $30 \mathrm{~ms}$ RV lead preactivation (VVD - 30) to $30 \mathrm{~ms} \mathrm{LV}$ lead preactivation (VVD 30) with intervals of $10 \mathrm{~ms}$ between pacing strategies. A third degree AV block was assumed for all the pacing scenarios. Pacing leads were positioned on the apical endocardium (RV lead) and on the lateral free wall epicardium (LV lead) as commonly done in CRT. A physiological activation (starting from the His bundle) was also simulated for each geometry for control purposes. We follow clinical guidelines regarding the positioning of the $\mathrm{LV}$ and $\mathrm{RV}$ lead. Therefore, we do not consider positioning the leads in other areas such as the His bundle that could be considered a better position in order to produce physiological activations. The reason is that the insulation that surrounds the Purkinje network plus the lack of Purkinje terminals in that area would prevent the electrical impulse to get inside the system ${ }^{23}$ and the re-synchronization will not succeed. The pacemaker lead stimulus was modeled as a $1 \mathrm{~mm}^{3}$ cube where transmembrane current of $0.05 \mu \mathrm{A} / \mathrm{cm}^{3}$ was injected for $1 \mathrm{~ms}$.

To further study the contribution of the PS to the activation sequence, all pacing simulations were repeated in absence of the PS. Two types of models were used: (i) models with physiological conductivity values in all myocardial tissue, and (ii) models with increased conductivity values on the endocardium ( $10 \%$ of the wall thickness on the whole ventricles). The increments in the conductivities at endocardium were adjusted to reach a conduction velocity of $2 \mathrm{~m} / \mathrm{s}$. With this velocity, the LV endocardial depolarization 
takes place in approximately $60 \mathrm{~ms}$, which is in the range of normal values. ${ }^{46}$

\section{Data Analysis}

Isochrone maps of local activation times (LATs) in the $3 \mathrm{D}$ models were used to study activation patterns during each simulation (Figs. 2 and 3). LAT was calculated as the time with respect to a reference (i.e., initial lead stimulus) at which the potential of a cell reached a threshold (depolarized). Isochrone maps depict the spatial distribution of LATs in $10 \mathrm{~ms}$ intervals.

Patterns observed for each pacing scenario and cardiac anatomy were analyzed with cumulative frequency histograms of the amount of activated LV myocardium, similarly as described by Shannon et al. ${ }^{36}$ Each histogram shows the percentage of LV myocardial tissue activated in time intervals over the depolarization sequence.

In each case, the LV was further analyzed by calculating the mean activation time for each region of the American Heart Association standard 17 segment division. Apart from the overall activation pattern that the histograms convey, it is important to consider the spatial order of activation and study whether the LV segments are properly synchronized. These data are important to search for the scenario that shows a better synchrony between the different walls from the point of view of the electrical activation.

\section{RESULTS AND DISCUSSION}

In the physiological simulations, the activation sequence was triggered from the AV node, which activates the His bundle. The right and left bundle branches propagate the activation to the PS. Since Purkinje fibers are isolated from the myocardium, they only stimulate the tissue through PMJs. The PS initiates as many activation wavefronts on the endocardial surface as there are PMJs on the network, giving rise to a rapid sequence of activation that propagates from apex to base and from endocardium to epicardium, in accordance with previously reported values. ${ }^{10,22}$ The time for all ventricular tissue to depolarize (total activation time; TAT) was computed using the first endocardial breakthrough as a starting point. The values were $90 \mathrm{~ms}$ for the healthy heart, $105 \mathrm{~ms}$ for HCM, and $119 \mathrm{~ms}$ for DCM.

In the case of stimulations triggered by a pacemaker, the activation sequence presented significant differences as compared to sinus activations. Figures 2 and 3 show the LATs in a cut plane displaying the LV's posterior wall for the DCM and HCM models in three different pacing scenarios, (a) VVD $-30 \mathrm{~ms}$, (b) VVD $0 \mathrm{~ms}$, (c) VVD $30 \mathrm{~ms}$, and their corresponding basal views (d)-(f). During ventricular pacing in models with PS, the propagation of excitation is slower and less uniform than during His stimulation. The stimulus given by the LV lead of the pacemaker gives rise to a wavefront that propagates through the myocardial tissue near the pacing site. After crossing the
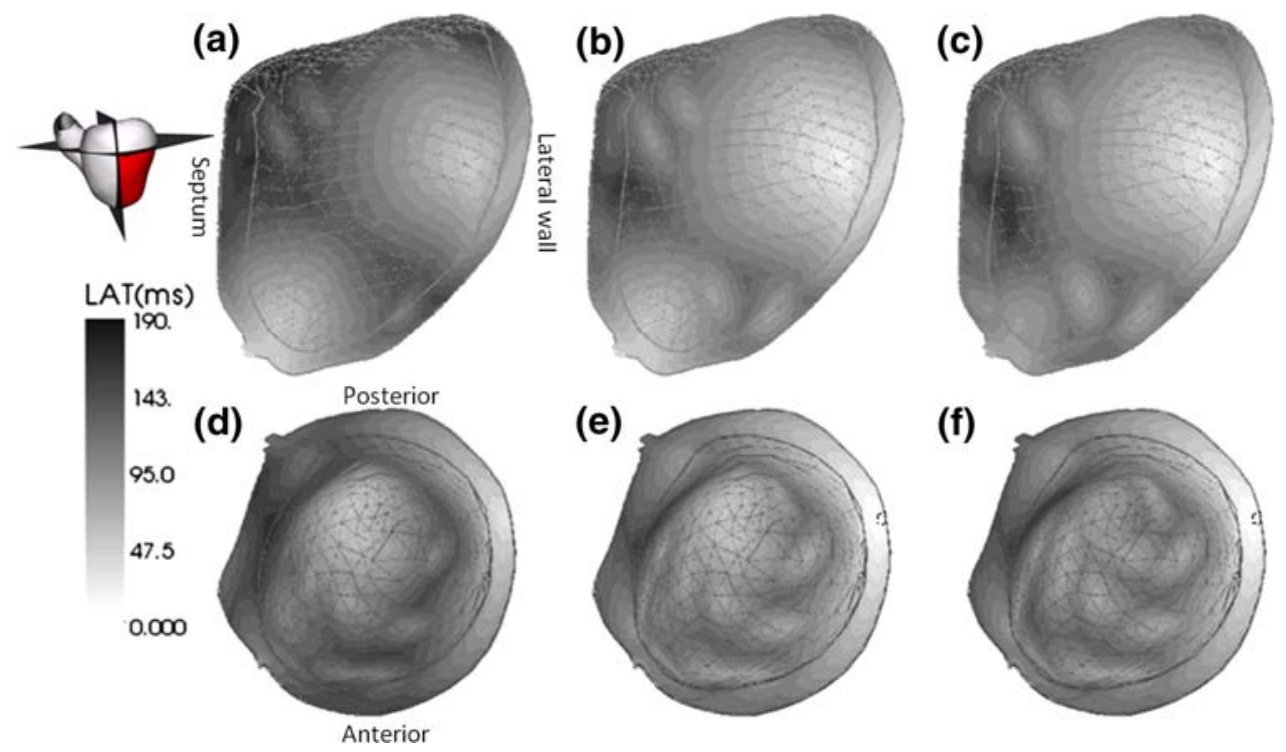

FIGURE 2. Local activation times (LATs) in DCM with PS for different pacing strategies. Anterior view of the LV's posterior wall showing the 3D isochronal maps of the LATs for three pacing scenarios, (a) VVD $-30 \mathrm{~ms}$, (b) VVD 0 ms, (c) VVD $30 \mathrm{~ms}$, and their corresponding basal views (d-f). 

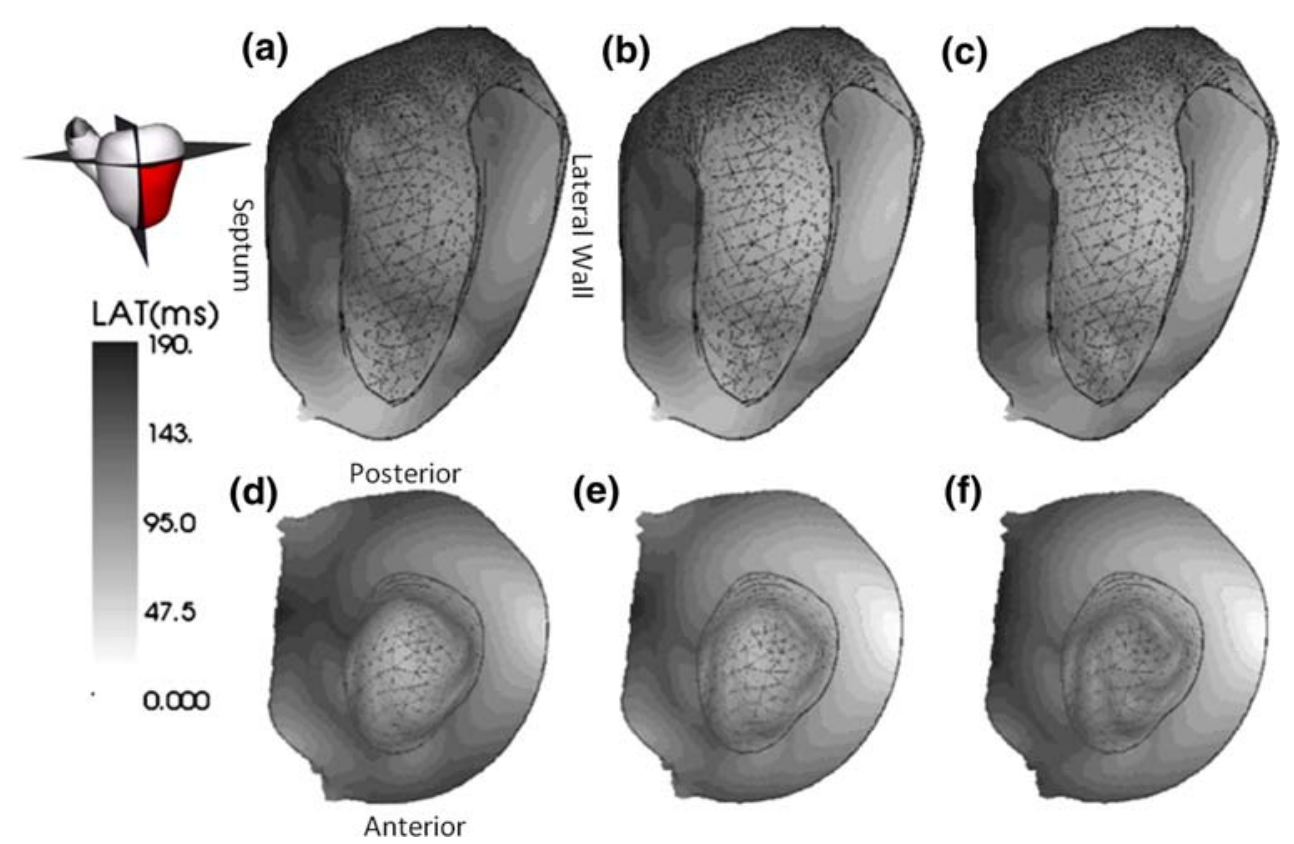

(e)

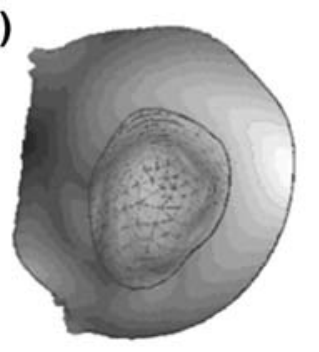

(f)

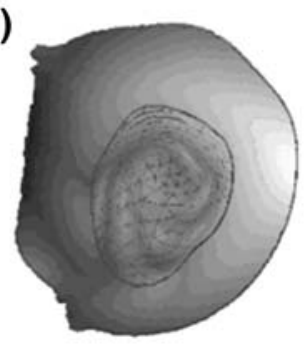

FIGURE 3. Local activation times in HCM with PS for different pacing strategies. Anterior view of the LV's posterior wall showing the 3D isochronal maps of the local activation times for three pacing scenarios, (a) VVD - $30 \mathrm{~ms}$, (b) VVD 0 ms, (c) VVD $30 \mathrm{ms,}$, and their corresponding basal views (d-f).

LV lateral wall, the wavefront retrogradely activates the closest PMJ on the endocardial surface. Once in the PS, the depolarization spreads rapidly, entering the bulk myocardium through other PMJs and rapidly activating remote areas. For the RV lead, which is placed on the thin-walled right endocardial apex near the PS, the onset of retrograde activation occurs quickly after the initial stimulus generating a wavefront that travels across the septum at different heights. It contributes to the septal activation, and it also heavily influences the LV's anterior and posterior wall in pacing strategies with VVD $-30 \mathrm{~ms}$ and VVD $0 \mathrm{~ms}$. Moreover, due to the proximity of the RV lead to the septal wall, the wavefront crosses the septum and contributes to the activation of the LV apex (see Figs. 2 and 3) and initiates retrograde activation of the PMJs on the LV endocardium. For the models with PS considered, this last effect was only observed for VVD $-30 \mathrm{~ms}$. In the other cases the LV PMJ closest to the RV lead generated breakthrough had already been activated by the LV lead. However, RV pacing in models with increased endocardial conductivity always caused retrograde activation of the LV apical endocardium as seen clinically. ${ }^{19}$ Consequently, the activation sequence highly depends on the wall thickness and PS distribution on the endocardial surface. Due to our assumption for the PS, for which we fitted a unique structure onto the three geometries, the number of PMJs per endocardial unit area varied between geometries. The HCM, with its smaller endocardial surface, had the highest PMJ density, while the DCM had the lowest. Figures 2 and 3 show the wavefront on the lateral wall crossing from epicardium to endocardium faster in the DCM than in the HCM model; however, once on the endocardium, both impulses have to propagate towards a PMJ and, given the density of these, the HCM's wavefront has a higher chance of finding one.

Figure 4 shows histograms of the percentage of activated LV tissue for three pacing scenarios. Simulations with VVD $-30 \mathrm{~ms}$ ( $\mathrm{a}, \mathrm{d}$, and $\mathrm{g}$ ) show a period of inactivity on the LV myocardium while the wavefront from the RV lead reaches the septal wall. On the remaining plots, activation always starts at $0 \mathrm{~ms}$, although this initial excitation is almost imperceptible on the plots because of the very slow rate of initial rise. As activation spreads, the associated curves start to drift apart. For simulations with an underlying PS (Figs. 4a-4c), a high slope represents the contribution of retrograde PS activation. Thus, the wall thickness and the distance to the closest PMJ determine the instant and rate of the major increase in slope on each curve. The LV PS is reached first in the healthy model (solid line), whereas in HCM (dotted) due to the thicker wall and in DCM (dashed line,) due to the lower density of PMJs, there is a higher delay. Simultaneous activation (VVD $0 \mathrm{~ms}$ ) produced the most uniform results.

Simulations using myocardial models with normal conductivity values, but lacking the PS take longer to activate (see Figs. 4d-4f). The main reason is that the 

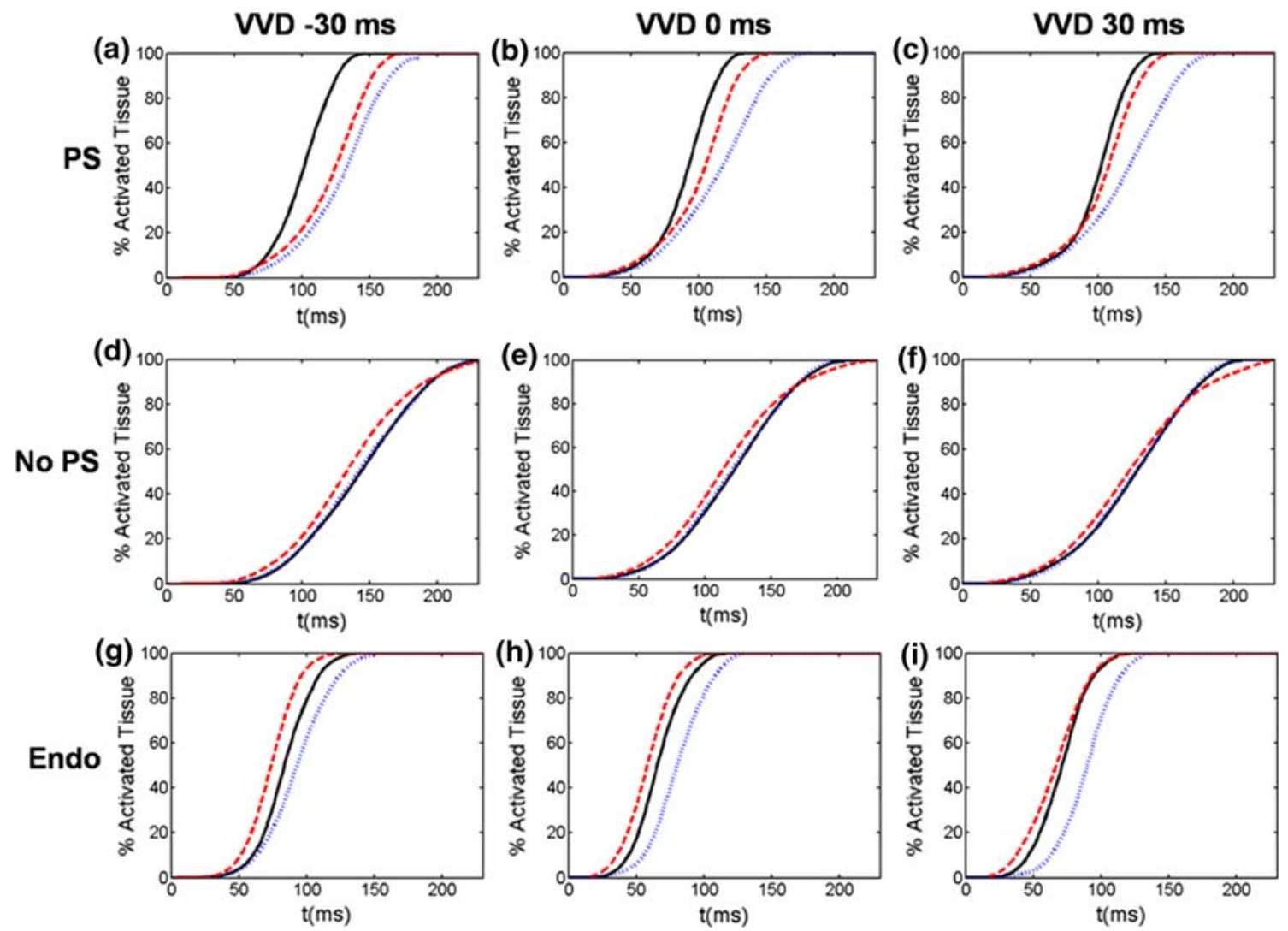

FIGURE 4. Cumulative frequency histograms of the normalized percentage of activated tissue. The lines correspond to: healthy (solid); DCM (dashed); and HCM (dotted) models. The first row corresponds to simulations with PS while the second and third rows correspond to simulations without the PS and with normal and increased endocardial conductivities, respectively.

wavefront has to reach remote areas traveling only through bulk myocardium. In Fig. 4f, the slowest in completing the activation of the LV was the DCM geometry $(230 \mathrm{~ms})$ the one with the largest circumferential distance to cover, while the Healthy and HCM geometries had finished by about $200 \mathrm{~ms}$. The shapes of the Healthy and HCM curves do not differ significantly.

Another example of the dependence of the activation sequence with the PS distribution is illustrated in Fig. 4c (VVD $30 \mathrm{~ms}$ ). In this figure, the curve representing the HCM model (blue; dotted) achieves its maximum slope later than the other geometries. This can be attributed to the fact that retrograde excitation in the HCM occurs later than in the healthy or DCM hearts. While the excitation wavefront is still propagating through the thick ventricular wall in the HCM model, it has already reached a PMJ on the endocardial side in the other two cases. Notably, the trajectory of this curve is nearly indistinguishable from the activation histogram for the HCM heart with no PS (Fig. 4f).

In Figs. $4 \mathrm{~g}-4 \mathrm{i}$, the curves correspond to models without PS but with increased endocardial conductivity.
This implies the assumption that every node on this layer behaves as a PMJ, explaining the very steep slopes of the curves. Moreover, it also implies that the retrograde activation for these models was only dependent on wall thickness. As a consequence, the DCM model, which has the thinnest ventricular wall, was always the fastest to depolarize (shortest TAT), followed by the Healthy and HCM models. Another aspect to note on these simulations is that the depolarization caused by the RV lead reached the LV apex at the endocardium and initiated rapid activation wavefronts that contributed greatly to LV depolarization. This effect is highlighted in the DCM curves, which tend to move closer to the Healthy curve as the pacing shifts from RV preactivation to LV preactivation (see the distance between the dashed and the solid line, which diminishes from Figs. $4 \mathrm{~g}$ to $4 \mathrm{i})$. Nevertheless, this modeling approach is fundamentally inaccurate, since PMJs are not homogeneously distributed with extremely high density on the endocardium. ${ }^{18,10}$ Therefore, the time required to retrogradely activate the PS is neglected.

The bullseye plots (see Fig. 5) correspond to VVD $0 \mathrm{~ms}$ with the possible combinations: the three 


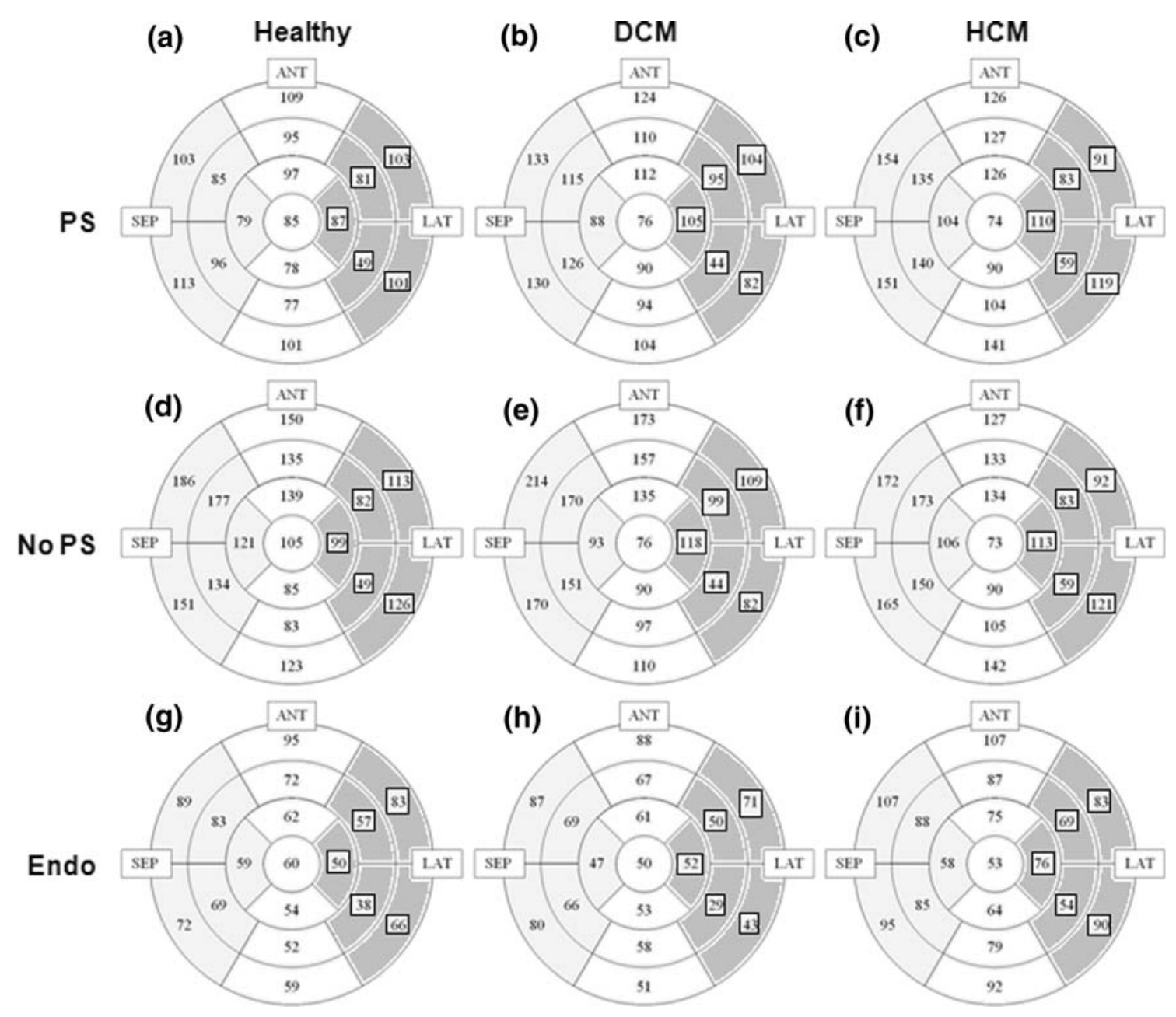

FIGURE 5. Bullseye plots of the AHA 17 segment division, showing LV activation times for simultaneous pacing (VVD 0 ms). The first row corresponds to simulations with PS while the second and third rows correspond to simulations without the PS and with normal and increased endocardial conductivities, respectively.

geometries with PS, no PS with normal conductivity values, and no PS with increased endocardial conductivities. Results from models with PS when compared to results from models with an absence of the PS and normal conductivity values indicate that corresponding geometries have very similar mean activation values for the lateral wall (pairs a-d, b-e, c-f). This highlights the role of the LV lead alone on this task. In contrast with lateral wall, the septal activation on these plots indicates a significant delay between corresponding models.

Intraventricular dyssynchrony is referred to as a marked delay of the onset of contraction between the septal and LV lateral wall. One potential treatment is a CRT device with the LV lead positioned at the site of longest delay. Therefore, the electrical activation as displayed on the bullseye plot conveys information that can be associated to intraventricular dyssynchrony. The analysis of activation sequences across geometries with PS (Figs. 5a-5c), shows the propagation wavefront on the septal wall always following an apex to base pattern. However, this pattern is lost for the lateral wall activation due to the action of the $\mathrm{LV}$ lead in the mid-posteriolateral wall. Moreover, in this region, the order of activation depends on the geometry. Within models lacking the PS but with normal conductivity values (Figs. $5 \mathrm{~d}-5 \mathrm{f}$ ) the apex to base activation pattern of the septal wall is preserved, although with a significant prolongation in activation compared to with-PS counterparts. This same pattern is observed in models with increased conductivity values (Figs. 5g-5i) which have the earliest onset and fastest depolarization of the LV's septal and lateral wall.

Our simulations show that the mean activation times of the septum and the lateral wall are more synchronous and physiological in models with PS (see Fig. 5) as compared to models without PS, which highlights the role of the PS in maintaining synchronicity between the 
walls of the ventricles by minimizing their activation delay. The models with normal conductivity values and no PS showed a prolonged activation on their histograms and an activation widely spread in time when compared to simulations with PS. TAT is associated to the QRS interval, an index that is used to assess the CRT optimization. ${ }^{16}$ Thus activated tissue histograms can be interpreted as a global index of intraventricular synchronicity. Simulations with lower intraventricular delays, as displayed on bullseye plots, showed a higher maximum slope and a reduction of TAT.

\section{Limitations of This Study}

The use of computer models to study and predict the response to specific therapies is still in an early stage. The uncertainty of basic physiological constants, coupled with the spatial heterogeneity, is a clear source of concern.

The ionic models used come from two different species. Since only the onset of depolarization was of interest, propagation velocity was of primary importance. To this end, sodium conductance in the PS was adjusted, and junctional parameters were tuned to properly model propagation across the PMJs. Differences in action potential duration between the two ionic models did not affect the results. For future studies involving repolarization, a more realistic human ionic model of Purkinje fibers should be implemented.

Even though we were able to show the importance of the PS in the electrical activation and its effect in CRT, the PS model needs to be improved. The density of terminals in the system is lower than it has been reported in histological studies, ${ }^{21}$ and therefore delays in the initial activation could be observed. The time to reach the PS from a lead can be affected by the density of terminals. A specific study to find out a more accurate density and ramification model of the PS remains to be done.

Invasive techniques, such as electrical mapping are among the few in vivo studies that can be used to validate the functioning of the heart electrical system. Although the data are sparse and difficult to interpret, a validation based on these recording remains to be done. This is a very challenging step that requires specific techniques in order to bring together electrical and geometrical properties of the heart. ${ }^{5}$

\section{CONCLUSION}

We have presented a cardiac electrical simulation study to assess the importance of anatomical and histological substructures on the paced heart. The computer model includes important substructures, such as the PS and myocardial fiber orientation based on a mathematical formulation. The branching Purkinje structure was approached by manual delineation following anatomical landmarks and its electrical behavior was modeled as a $1 \mathrm{D}$ cable network. ${ }^{41}$ The model is able to simulate the electrical activation produced by a BV pacemaker and reproduces the effect of retrograde activation at PMJs.

The results showed that the use of a detailed and sophisticated PS is vital to account in simulations with CRT. Its retrograde conduction property is important to determine the synchronous activation between the LV walls. The usage of models without a PS can overestimate the degree of intraventricular dyssynchrony.

Although while pacing from the epicardium it is not possible to reproduce a physiological pattern, it can however maintain the most electrophysiological resemblance (apex to base) depending on the pathological structure. Applying the same pacing strategy to different models elucidates important local variations, made manifest by differences in activation pattern. Between geometries for a given VVD, the most notable effect on the activation pattern is seen on the lateral wall, where the apical and basal segments changed their order of activation. This should be considered in clinical practice at the moment of the LV lateral wall lead positioning to improve the outcome of the procedure. Further work on simulations should test different lead positions to achieve this goal.

\section{ACKNOWLEDGMENTS}

This research has been partially funded by the Industrial and Technological Development Center (CDTI) under the CENIT Programme (CDTEAM Project) and the European Community's Seventh Framework Programme (FP7/2007-2013) under grant agreement no. 224495 (euHeart Project). Dr. R. Sebastian was funded by the Ministerio de Ciencia e Innovacion (Juan de la Cierva program). Dr. B. Bijnens is an ICREA Research Professor at Universitat Pompeu Fabra. Dr. E. Vigmond and P. Boyle were funded by The Natural Sciences and Engineering Research Council of Canada. P. Boyle is supported by the Alberta Ingenuity Fund.

\section{REFERENCES}

${ }^{1}$ Abraham, W. T., Rationale and design of a randomized clinical trial to assess the safety and efficacy of cardiac 
resynchronization therapy in patients with advanced heart failure: the Multicenter Insync Randomized Clinical Evaluation (MIRACLE), J. Card Fail. 6(4):369-380, 2000. ${ }^{2}$ Boyle, P., M. Deo, and E. Vigmond. Behaviour of the Purkinje system during defibrillation-strength shocks. In: Proc. 29th Annual International Conference of the IEEE Engineering in Medicine and Biology Society EMBS, 2007, pp. 419-422.

${ }^{3}$ Camm, A. J., T. F. Lüscher, and P. Serruys, Eds., The ESC Textbook of Cardiovascular Medicine. Wiley-Blackwell Publishing, Oxford, UK, 2006.

${ }^{4}$ Castellant, P., M. Fatemi, E. Orhan, Y. Etienne, and J. J. Blanc, Patients with non-ischaemic dilated cardiomyopathy and hyper-responders to cardiac resynchronization therapy: characteristics and long-term evolution, Europace 11:350-355, 2009.

${ }^{5}$ Cazeau, S., C. Alonso, G. Jauvert, A. Lazarus, and P. Ritter, Cardiac resynchronization therapy, Europace, 5(Suppl 1):S42-S48, 2004.

${ }^{6}$ Chung, E. S., A. R. Leon, L. Tavazzi, J.-P. Sun, P. Nihoyannopoulos, J. Merlino, W. T. Abraham, S. Ghio, C. Leclercq, J. J. Bax, C.-M. Yu, J. Gorcsan, M. S. J. Sutton, J. D. Sutter, and J. Murillo, Results of the predictors of response to CRT (PROSPECT) trial, Circulation, 117(20):2608-2616, 2008.

${ }^{7}$ Clerc, L., Directional differences of impulse spread in trabecular muscle from mammalian heart, J. Physiol. 255(2):335-346, 1976.

${ }^{8}$ Colli-Franzone, P., L. Guerri, M. Pennachio, and B. Taccardi, Spread of excitation in 3-d models of the anisotropic cardiac tissue. II. Effects of fiber architecture and ventricular geometry, Math. Biosci. 147:131-171, 1998.

${ }^{9}$ DiFrancesco, D., and D. Noble. A model of cardiac electrical activity incorporating ionic pumps and concentration changes. Philos. Trans. R Soc. Lond. B Biol. Sci. 307(1133): 353-398, 1985.

${ }^{10}$ Durrer, D., R. T. van Dam, G. E. Freud, M. J. Janse, F. L. Meijler, and R. C. Arzbaecher. Total excitation of the isolated human heart. Circulation 41(6):899-912, 1970.

${ }^{11}$ Fenton, F. H., E. M. Cherry, A. Karma, and W.-J. Rappel, Modeling wave propagation in realistic heart geometries using the phase-field method, Chaos, 15(1):13502, 2005.

${ }^{12}$ Frangi, A. F., D. Rueckert, J. A. Schnabel, and W. J. Niessen, Automatic construction of multiple-object three-dimensional statistical shape models: application to cardiac modeling, IEEE Trans. Med. Imaging, 21(9):1151-1166, 2002.

${ }^{13}$ Gassis, S., and A. R. León, Cardiac resynchronization therapy: strategies for device programming, troubleshooting and follow-up, J. Interv. Card Electrophysiol. 13(3): 209-222, 2005.

${ }^{14}$ Henriquez, C., and A. Papazoglou, Using computer models to understand the roles of tissue structure and membrane dynamics in arrhythmogenesis, Proc. IEEE, 84(3):334-354, 1996.

${ }^{15}$ Jouk, P.-S., A. Mourad, V. Milisic, G. Michalowicz, A. Raoult, D. Caillerie, and Y. Usson, Analysis of the fiber architecture of the heart by quantitative polarized light microscopy. accuracy, limitations and contribution to the study of the fiber architecture of the ventricles during fetal and neonatal life. Eur. J. Cardiothorac. Surg. 31(5):915921, 2007.

${ }^{16}$ Kamireddy, S., S. K. Agarwal, E. Adelstein, S. Jain, and S. Saba. Correlation of electrical and mechanical reverse remodeling after cardiac resynchronization therapy. Ann. Noninvasive Electrocardiol. 14(2):153-157, 2009.
${ }^{17}$ Kerckhoffs, R. C. P., P. H. M. Bovendeerd, F. W. Prinzen, K. Smits, and T. Arts, Intra- and interventricular asynchrony of electromechanics in the ventricularly paced heart, J. Eng. Math. 47:201-216, 2003.

${ }^{18}$ Kerckhoffs, R. C. P., O. P. Faris, P. H. M. Bovendeerd, F. W. Prinzen, K. Smits, E. R. McVeigh, and T. Arts, Timing of depolarization and contraction in the paced canine left ventricle: Model and experiment, J. Cardiovasc. Electrophysiol., 14:S188-S195, 2003.

${ }^{19}$ Kimmel, M. W., N. D. Skadsberg, C. L. Byrd, D. J. Wright, T. G. Laske, and P. A. Iaizzo. Singlesite ventricular and biventricular pacing: investigation of latest depolarization strategy. Europace 9:1163-1170, 2007.

${ }^{20}$ MacGowan, G. A., E. P. Shapiro, H. Azhari, C. O. Siu, P. S. Hees, G. M. Hutchins, J. L. Weiss, and F. E. Rademakers, Noninvasive measurement of shortening in the fiber and cross-fiber directions in the normal human left ventricle and in idiopathic dilated cardiomyopathy. Circulation 96:535-541, 1997.

${ }^{21}$ Massing, G. K., and T. N. James. Anatomical configuration of the his bundle and bundle branches in the human heart. Circulation 53(4):609-621, 1976.

${ }^{22}$ Myerburg, R. J., H. Gelband, K. Nilsson, A. Castellanos, A. R. Morales, and A. L. Bassett. The role of canine superficial ventricular muscle fibers in endocardial impulse distribution. Circ. Res. 42(1):27-35, 1978.

${ }^{23}$ Myerburg, R. J., K. Nilsson, and H. Gelband. Physiology of canine intraventricular conduction and endocardial excitation. Circ. Res. 30(2):217-243, 1972.

${ }^{24}$ Nanthakumar, K., J. Jalife, S. Masse, E. Downar, M. Pop, J. Asta, H. Ross, V. Rao, S. Mironov, E. Sevaptsidis, J. Rogers, G. Wright, and R. Dhopeshwarkar, Optical mapping of langendorff-perfused human hearts: establishing a model for the study of ventricular fibrillation in humans, Am. J. Physiol. Heart Circ. Physiol., 293:H875880, 2007.

${ }^{25}$ Noble, D., From the hodgkin-huxley axon to the virtual heart, J. Physiol., 580(Pt 1):15-22, 2007.

${ }^{26}$ Noble, D., and Y. Rudy, Models of cardiac ventricular action potentials: Iterative interaction between experiment and simulation, Philos. Trans. Math. Phys. Eng. Sci., 359(1783):1127-1142, 2001.

${ }^{27}$ Ordas, S., E. Oubel, R. Sebastian, and A. F. Frangi, Computational atlas of the heart. In: Proc. 15th International Symposium on Image and Signal Processing and Analysis (ISPA), 2007, pp. 338-342.

${ }^{28}$ Plank, G., M. Liebmann, R. W. dos Santos, E. J. Vigmond, and G. Haase. Algebraic multigrid preconditioner for the cardiac bidomain model. IEEE Trans. Biomed. Eng. 54(4): 585-596, 2007.

${ }^{29}$ Plonsey, R. Bioelectric sources arising in excitable fibers (alza lecture). Ann. Biomed. Eng., 16(6):519-546, 1988.

${ }^{30}$ Pollard, A. E., M. J. Burgess, and K. W. Spitzer, Computer simulations of three-dimensional propagation in ventricular myocardium. effects of intramural fiber rotation and inhomogeneous conductivity on epicardial activation, Circ. Res. 72(4):744-756, 1993.

${ }^{31}$ Potse, M., B. Dub, J. Richer, A. Vinet, and R. M. Gulrajani, A comparison of monodomain and bidomain reaction-diffusion models for action potential propagation in the human heart, IEEE Trans. Biomed. Eng., 53(12 Pt 1): 2425-2435, 2006.

${ }^{32}$ Ramanathan, C., P. Jia, R. Ghanem, K. Ryu, and Y. Rudy, Activation and repolarization of the normal 
human heart under complete physiological conditions. Proc. Natl. Acad. Sci. USA, 103(16):6309-6314, 2006.

${ }^{33}$ Reumann, M., D. Farina, R. Miri, S. Lurz, B. Osswald, and O. Dössel, Computer model for the optimization of av and vv delay in cardiac resynchronization therapy, Med. Biol. Eng. Comput. 45(9)845-854, 2007.

${ }^{34}$ Rinaldi, C. A., C. A. Bucknall, and J. S. Gill, Beneficial effects of biventricular pacing in a patient with hypertrophic cardiomyopathy and intraventricular conduction delay, Heart 87(6):e6, 2002.

${ }^{35}$ Rodríguez, B., L. Li, J. C. Eason, I. R. Efimov, and N. A. Trayanova, Differences between left and right ventricular chamber geometry affect cardiac vulnerability to electric shocks, Circ. Res. 97(2)168-175, 2005.

${ }^{36}$ Shannon, J., C. O. Navarro, T. McEntee, G. Riddell, J. A. Adgey, and E. W. Lau. An early phase of slow myocardial activation may be necessary in order to benefit from cardiac resynchronization therapy. J. Electrocardiol. 41:531-535, 2008.

${ }^{37}$ Streeter, D. Gross morphology and fiber geometry of the heart. In: Handbook of Physiology, The Cardiovascular System, vol. 1, edited by R. Berne. Bethesda, USA: American Physiological Society, 1979, pp. 61-112.

${ }^{38}$ Ten Tusscher, K. H. W. J., and A. V. Panfilov, Cell model for efficient simulation of wave propagation in human ventricular tissue under normal and pathological conditions, Phys. Med. Biol., 51(23): 6141-6156, 2006.
${ }^{39}$ Ten Tusscher, K. H. W. J., and A. V. Panfilov, Modelling of the ventricular conduction system, Prog. Biophys. Mol. Biol. 96:152-170, 2008.

${ }^{40}$ Tezuka, F. The morphogenesis of left ventricular hypertrophy of heart as correlated with the muscle fiber orientation. In: Proc. of the First International Symposium of Science on Form, edited by Y. Kato, R. Takaki, and J. Toriwaki. Tokyo: KTK Scientific Publishers, 1986, pp. 579-584.

${ }^{41}$ Vigmond, E. J., and C. Clements. Construction of a computer model to investigate sawtooth effects in the Purkinje system. IEEE Trans. Biomed. Eng., 54(3):389-399, 2007.

${ }^{42}$ Vigmond, E. J., C. Clements, D. M. McQueen, and C. S. Peskin, Effect of bundle branch block on cardiac output: a whole heart simulation study. Prog. Biophys. Mol. Biol., 97(2-3):520-542, 2008.

${ }^{43}$ Vigmond, E. J., R. W. dos Santos, A. J. Prassl, M. Deo, and G. Plank, Solvers for the cardiac bidomain equations, Prog. Biophys. Mol. Biol. 96(1-3):3-18, 2008.

${ }^{44}$ Vigmond, E. J., M. Hughes, G. Plank, and L. J. Leon. Computational tools for modeling electrical activity in cardiac tissue. J. Electrocardiol. 36(Suppl):69-74, 2003.

${ }^{45}$ Whiteley, J. P. An efficient numerical technique for the solution of the monodomain and bidomain equations. IEEE Trans. Biomed. Eng. 53(11):2139-2147, 2006.

${ }^{46}$ Yu, C.-M., D. L. Hayes, and A. Auricchio, Eds., Cardiac Resynchronization Therapy. Blackwell Publishing, Oxford, UK, 2006. 